\title{
EDGE-NEIGHBor RuPTURE DEgREE ON GRAPH OPERATIONS
}

\author{
Saadet Eskiizmirliler ${ }^{1}$, Zeynep Örs Yorgan cioğlu ${ }^{2}$, Refet Polat ${ }^{3}$ \\ And Mehmet Ümit Gürsoy ${ }^{4}$ \\ ${ }^{1,3}$ Department of Mathematics, Faculty of Science, Yasar University, Izmir, Turkey, \\ ${ }^{2}$ Maritime and Port Management Program, Vocational School, \\ Yasar University, Izmir, Turkey, \\ ${ }^{4}$ Izmir, Turkey,
}

\begin{abstract}
Vulnerability and reliability parameters measure the resistance of the network to disruption of operation after the failure of certain stations or communication links in a communication network. An edge subversion strategy of a graph $G$, say $S$, is a set of edge $(s)$ in Gwhose adjacent vertices which is incident with the removal edge(s) are removed from $G$. The survival subgraph is denoted by $G-S$. The edgeneighbor-rupture degree of connected graph $G, E N R(G)$, is defined to beENR $(G)=\max \{\omega(G-S)-$ $|S|-m(G-S): S \subseteq E(G), \omega(G-S) \geq 1\}$ where Sis any edge-cut-strategy of $G, \omega(G-S)$ is the number of the components of $G-S$, and $m(G-S)$ is the maximum order of the components of $G-S$. In this paper we give some results for the edge-neighbor-rupture degree of the graph operations and Thorny graph types are examined.
\end{abstract}

\section{KEYWORDS}

Edge-neighbor-rupture degree, Thorny graphs, Vulnerability, Reliability.

\section{INTRODUCTION}

A communication network can be brokedown to pieces partially or completely from unexpected factors. This situation can prevent data transmit so there would be a big problem on the system to perform it's task. Therefore, the vulnerability and the reliability measure the resistance of the network disturbance of operations after the failure of certain stations. To measure the vulnerability and the reliability we have some parameters which are connectivity $[7,11,12]$, integrity [3], scattering number [8], rupture degree [9], neighbor-rupture degree [1] and edgeneighbor-rupture degree [2].

Terminology and notations are not defined in this paper but it can be found [4,5]. Let $G=$ $(V, E)$ be a simple graph and let $e$ be any edge of $G$. The set, $N(e)=\{f \in E(G) \mid e \neq f ; e$ and $f$ are adjacent $\}$ is the open neighborhood of $e$, and $[e]=\{e\}$ $U$ (e) is the closed neighborhood of $e$. An edge $e$ in Gis said to be subverted if the closed neighborhood of $e$ is removed from $G$. In other words, if $e=\{u, v\} \operatorname{than} G-[\mathrm{e}]=G-\{u, v\}$. A set of edges $S=\left\{e_{1}, e_{2}, \ldots, e_{m}\right\}$ is called an edge subversion strategy of $G$ if each of the edges in $S$ has been subverted from $G$. If $S$ has been subverted from the graph $G$, then the remaining graph is called survival graph, denoted by $G-S$. An edge subversion strategy $S$ is called an edge-cutstrategy of $G$ if the survival subgraph $G-S$ is disconnected or is a single vertex or the empty graph 
Applied Mathematics and Sciences: An International Journal (MathSJ ), Vol. 5, No. 1/2/3, September 2018

[10].Sis any edge-cut-strategy of $G,(G-S)$ is the number of the components of $G-S$, and $(G-S)$ is the maximum order of the components of $G-S$.

The definition of edge-neighbor-rupture degree of a connected graph $G$ is

$E N R(G)=\max \{\omega(G-S)-|S|-m(G-S): S \subseteq E(G), \omega(G-S) \geq 1\}$

Definition 1.1: $r \in N^{+}$and $\forall v \in \mathrm{V}(G)$ for each vertex of $G$, if $\operatorname{deg}(v)=r$ than $G$ is called $r$ regular graph [4].

Definition 1.2: $G_{1}$ and $G_{2}$ graphs have disjoint vertex sets $V_{1}$ and $V_{2}$ and edge sets $E_{1}$ and $E_{2}$ respectively. The join operation of two $G_{1}$ and $G_{2}$ graphs is denoted by $G_{1}+G_{2}$ and consist of $G_{1} \cup G_{2}$ which is union of two $G_{1}$ and $G_{2}$ graphs and all edges joining $V_{1}$ and $V_{2}$ [4].

Definition 1.3: Let $p_{1}, p_{2}, \ldots, p_{n}$ be non-negative integers. The Thorny Graph of the graph $G$, with parameters $p_{1}, p_{2}, \ldots, p_{n}$, is obtained by attaching $p_{i}$ new vertices of degree one to the vertex $u_{i}$ of the $\operatorname{graph} G, i=1,2, \ldots, n$.

The Thorny graph of the graph $G$ is denoted by $G^{\star}$, or if the respectiveparameters need to be specified, by $G^{\star}\left(p_{1}, p_{2}, \ldots, p_{n}\right)[6]$.

Definition 1.4:Forthree or more disjoint graphs $G_{1}, G_{2}, \ldots, G_{n}$, the sequential join

$G_{1}+G_{2}+\cdots+G_{n}$

is the graph

$\left(G_{1}+G_{2}\right) \cup\left(G_{2}+G_{3}\right) \cup \ldots \cup\left(G_{n-1}+G_{n}\right)[4]$.

Definition 1.5: Connectivity $k(G)$ is the minimum number of vertices that need to be removed in order to disconnect a graph [4].

Definition 1.6: The integrity of a graph $G=(V, E)$ is defined by $I(G)=\min \{|S|+m(G-$ $S)$ \}; $S \subset V(G)$ where $m(G-S)$ denotes the order of largest component in $G-S[3]$.

Definition 1.7: The rupture degree of a non-complete connected graph $G$ is defined by $r(G)=$ $\max \{\omega(G-S)-|S|-m(G-S): S \subset V(G), \omega(G-S)>1\}$ where $\omega(G-S)$ denotes the number of components in the graph $G-S$ and $m(G-S)$ is the order of the largest component of $G-S[9]$.

Definition 1.8: The neighbor integrity of a graph $G$ is defined by

$N I(G)=\min \{|S|+c(G-S): S \subset V(G)\}$ where $S$ is any vertex subversion strategy of $G$ and $c(G-S)$ is the order of the largest component of $G-S[10]$.

Definition 1.9: The neighbor rupture degree of a non-complete connected graph $G$ is defined to be $N r(G)=\max \{\omega(G-S)-|S|-c(G-S): S \subset V(G), \omega(G-S) \geq 1\}$ where $S$ is any vertex subversion strategy of $G, \omega(G-S)$ is the number of connected components in $G-S$ and $c(G-S)$ is the maximum order of the components of $G-S[13]$.

Definition 1.10: The edge-neighbor-rupture degree of a connected graph $G$ is defined to be $\operatorname{ENR}(G)=\max \{\omega(G-S)-|S|-m(G-S): S \subseteq E(G), \omega(G-S) \geq 1\}$, where $S$ is any edge- 
Applied Mathematics and Sciences: An International Journal (MathSJ ), Vol. 5, No. 1/2/3, September 2018 cut-strategy of $G, \omega(G-S)$ is the number of the components of $G-S$, and $m(G-S)$ is the maximum order of the components of $G-S$.

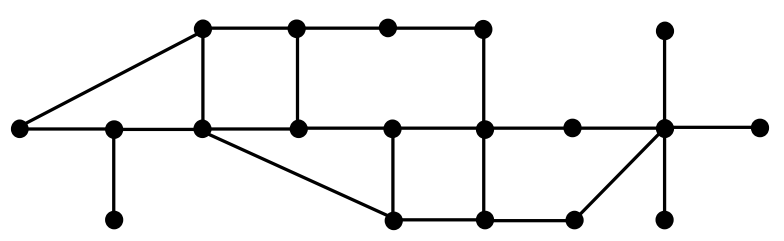

Example:Let's give an exampleof the calculation of the edge-neighborrupture-degree.

$\omega(G-S)=1$

$|S|=0$

$m(G-S)=19$

$\omega(G-S)-|S|-m(G-S)$

Figure 1.1

$$
=1-0-19=-18
$$

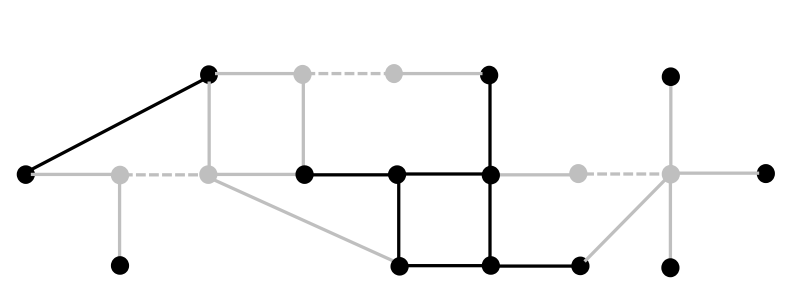

$\omega(G-S)=6$

$|S|=3$

$m(G-S)=7$

$\omega(G-S)-|S|-m(G-S)=6-3-7$

$$
=-4
$$

Figure 1.2

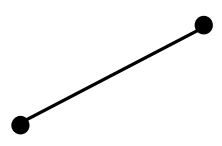

○
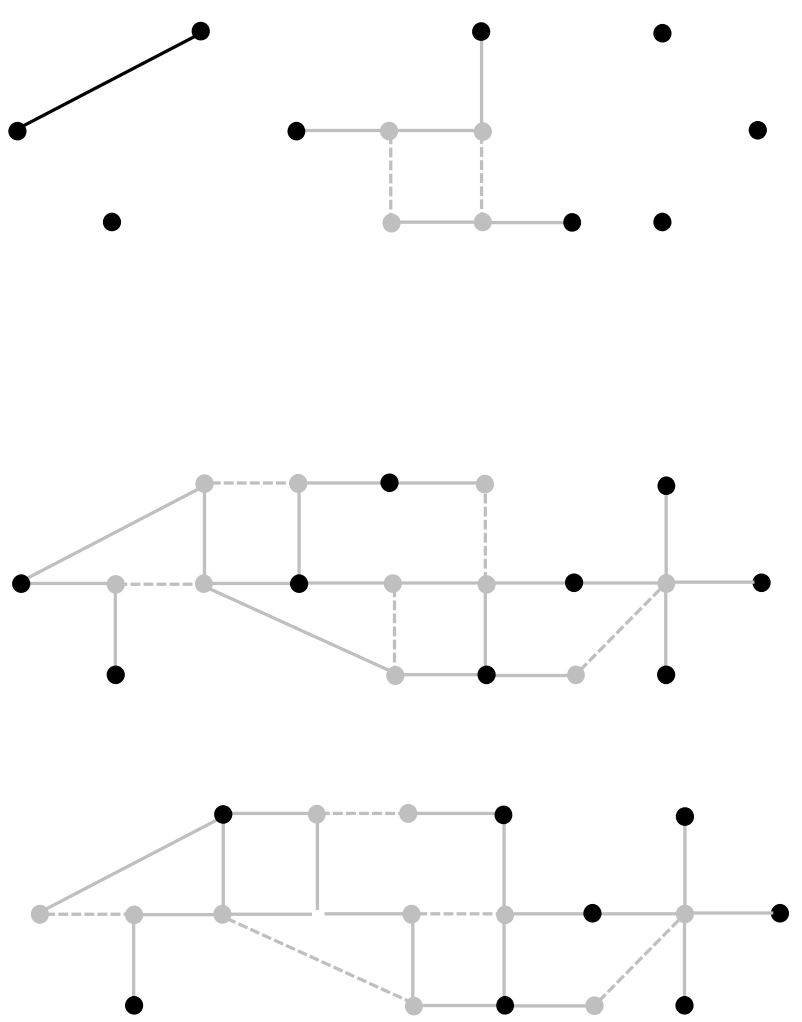

$\omega(G-S)=8$

$|S|=5$

$m(G-S)=2$

$\omega(G-S)-|S|-m(G-S)=8-5-2$

$=1$

Figure 1.3

$\omega(G-S)=9$

$|S|=5$

$m(G-S)=1$

$\omega(G-S)-|S|-m(G-S)=9-5-1$
$=3$

Figure 1.4

$\omega(G-S)=9$

$|S|=5$

$m(G-S)=1$

$\omega(G-S)-|S|-m(G-S)=9-5-$ $1=3$

Figure 1.5 
Applied Mathematics and Sciences: An International Journal (MathSJ ), Vol. 5, No. 1/2/3, September 2018

As shown, figure 1.1, figure 1.2, figure 1.3,figure 1.4 and figure 1.5

$\operatorname{ENR}(G)=\max \{-18,-4,1,3,3\}$

$\operatorname{ENR}(G)=3$

In this paper, the edge-neighbor-rupture degree of some graphs is obtained and the relations between edge-neighbor-rupture degree and other parameters are determined [2].

\section{EDGE-NEIGHBOR-RUPTURE DEgREe ON GRAPH OPERATIONS}

In this section some theorems are given for edge-neighbor-rupture degree on the graph operations. Connected, undirected, simple graphs are examined.

Theorem 2.1:Let $G$ be a regular graph and $G^{\star}$ is a thorny graph of $G$ (adding a vertex to any vertex of a graph). Then the edge-neighbor-rupture degree of $G$ is,

$\operatorname{ENR}\left(G^{\star}\right)=\operatorname{ENR}(G)+1$

Proof:Since $G$ is regular graph, you can start from any edge to delete. There are two cases.

Case 1: If we start to delete an edge that is incident to an added vertex, while Sedge-cutstrategynumber is not changing, $(G-S)$ numbers of components are increased 1 . So the result is increased 1.

Case 2:If we start to delete an edge that is not incident to an added vertex, while Sedge-cutstrategy number and the number of the components are not changed, maximum order of the components are increased. So the result is increased.

The result takes maximum value in case 1 . So the proof is completed.

Theorem 2.2: Edge-neighbor-rupture degree of thorny of regular graph $G$ (adding $i$ vertices equally to every vertex of a graph $G$ for $1 \leq i \leq n$ ) is,

$\operatorname{ENR}\left(G^{\star}\right)=n i-\left\lceil\frac{n}{2}\right\rceil-1$.

Proof: Let $S$ be anedge-cut-strategyof $G^{\star}$ and let $|S|=r$. There are two cases for the elements of $S$.

Case 1: If $0 \leq r<\left\lceil\frac{n}{2}\right\rceil$, then $\omega\left(G^{\star}-S\right) \leq 2 r i+1$ and $m\left(G^{\star}-S\right) \geq i+1$, so we have $\omega\left(G^{\star}-S\right)-|S|-m\left(G^{\star}-S\right) \leq 2 r i+1-r-(i+1)=2 r i-r-i$.

Let's $(r)=2 r i-r-i$. Since $f(r)$ is an increasing function in $0 \leq r<\left\lceil\frac{n}{2}\right]$, it takes its maximum value at $\left(\left\lceil\frac{n}{2}\right\rceil-1\right)$, and

$$
f\left(\left\lceil\frac{n}{2}\right\rceil-1\right)=2\left(\left\lceil\frac{n}{2}\right\rceil-1\right) i-\left\lceil\frac{n}{2}\right\rceil-i
$$


Applied Mathematics and Sciences: An International Journal (MathSJ ), Vol. 5, No. 1/2/3, September 2018

Thus we get $\left(G^{\star}\right) \leq 2\left(\left\lceil\frac{n}{2}\right\rceil-1\right) i-\left\lceil\frac{n}{2}\right\rceil-i$.

Case 2: If $\left\lceil\frac{n}{2}\right\rceil \leq r \leq \frac{3 n^{2}-n}{2}$, then $\omega\left(G^{\star}-S\right) \leq n i$ and $m\left(G^{\star}-S\right) \geq 1$, so we have

$\omega\left(G^{\star}-S\right)-|S|-m\left(G^{\star}-S\right) \leq n i-r-1$.

Let's $f(r)=$ in $-r-1$. Since $f(r)$ is a decreasing function in $0 \leq r<\left\lceil\frac{n}{2}\right\rceil$, it takes its maximum

value at $\left\lceil\frac{n}{2}\right\rceil$, and $f\left(\left\lceil\frac{n}{2}\right\rceil\right)=n i-\left\lceil\frac{n}{2}\right\rceil-1$

Thus we get $\operatorname{ENR}\left(G^{\star}\right) \leq n i-\left\lceil\frac{n}{2}\right\rceil-1$.

From Case 1 and Case 2 we have

$$
\operatorname{ENR}\left(G^{\star}\right) \leq n i-\left\lceil\frac{n}{2}\right\rceil-1 .
$$

There exist $S^{*}$ such that

$$
\begin{gathered}
r=\left\lceil\frac{n}{2}\right\rceil, \omega\left(G^{\star}-S^{*}\right)=n i \text { and } m\left(G^{\star}-S^{*}\right)=1, \text { thus we have } \\
\operatorname{ENR}\left(G^{\star}\right) \geq n i-\left\lceil\frac{n}{2}\right\rceil-1 .
\end{gathered}
$$

From (1) and (2) we get

$$
\operatorname{ENR}\left(G^{\star}\right)=n i-\left\lceil\frac{n}{2}\right\rceil-1 .
$$

Corollary 2.1: Let $P_{n}^{\star}$ is a Thorny graph of $P_{n}$ (adding $i$ vertices equally to every vertex of a graph for $\leq i \leq n)$. Then the edge-neighbor-rupture degree of $P_{n}^{\star}$ is,

$\operatorname{ENR}\left(P_{n}^{\star}\right)=n i-\left\lceil\frac{n}{2}\right\rceil-1$

Proof: It is the same as proof of Theorem 2.2

Corollary 2.2: Let $W_{n}^{\star}$ is a Thorny graph of $W_{n}$ (adding $i$ vertices equally to every vertex of a graph for2 $\leq i \leq n)$. Then the edge-neighbor-rupture degree of $W_{n}^{\star}$ is,

$\operatorname{ENR}\left(W_{n}^{\star}\right)=n i-\left\lceil\frac{n}{2}\right\rceil-1$.

Proof: It is the same as proof of Theorem 2.2.

Theorem 2.3: Edge-neighbor-rupture degree of Thorny graphof $S_{n}$ is $(i \geq 2)$,

$\operatorname{ENR}\left(S_{n}^{\star}\right)=n i-n$

Proof: Let $S$ be an edge-cut-strategyof $S_{n}^{\star}$ and let $|S|=r$. There are two cases for the elements of $S$. 
Applied Mathematics and Sciences: An International Journal (MathSJ ), Vol. 5, No. 1/2/3, September 2018

Case 1: If $0 \leq r<n-1$, then $\omega\left(S_{n}^{\star}-S\right) \leq r i+1$ and $m\left(S_{n}^{\star}-S\right) \geq i+1$, so we have

$\omega\left(S_{n}^{\star}-S\right)-|S|-m\left(S_{n}^{\star}-S\right) \leq r i+1-r-(i+1)=r i-r-i$.

Let $f(r)=r i-r-i$. Since $f(r)$ is an increasing function in $0 \leq r<n-1$, it takes its maximum value $(n-2)$ and $f(n-2)=(n-2)(i-1)-i$.

Thus we get $E N R\left(S_{n}^{\star}\right) \leq n i-n-3 i+2$.

Case 2: If $n-1 \leq r \leq n^{2}+n-1$ then $\omega\left(S_{n}^{\star}-S\right) \leq$ in and $m\left(S_{n}^{\star}-S\right) \geq 1$, so we have $\omega\left(S_{n}^{\star}-S\right)-|S|-m\left(S_{n}^{\star}-S\right) \leq n i-r-1$.

Let $f(r)=n i-r-1$. Since $f(r)$ is a decreasing function in $n-1 \leq r \leq n^{2}+n-1$ it takes its maximum value at $(n-1)$ and

$f(n-1)=n i-n+1-1=n i-n$.

Thus we get $\operatorname{ENR}\left(S_{n}^{\star}\right) \leq n i-n$.

From Case 1 and Case 2 we have

$$
\operatorname{ENR}\left(S_{n}^{\star}\right) \leq n i-n
$$

There exist $S^{*}$ such that

$r=n-1, \omega\left(S_{n}^{\star}-S^{*}\right)=n i$ and $m\left(S_{n}^{\star}-S^{*}\right)=1$, thus we have

$$
\operatorname{ENR}\left(S_{n}^{\star}\right) \geq n i-n
$$

From (3) and (4) we get $\left(S_{n}^{\star}\right)=n i-n$.

Theorem 2.4: Let $P_{n}, P_{m}$ is a path of order $n$ and $m$ respectively. The edge-neighbor-rupture degree of addition of $P_{n}$ and $P_{m}$ is,

$\operatorname{ENR}\left(P_{n}+P_{m}\right)=\left\{\begin{array}{rl}\operatorname{ENR}\left(P_{m}\right)-\frac{n}{2}, & n \text { is even } \\ \operatorname{ENR}\left(P_{m-1}\right)-\left\lceil\frac{n}{2}\right\rceil, & n \text { is odd }\end{array}, \quad n<m\right.$

\section{Proof:}

Case 1:If we select an edge-cut-strategy from $P_{n}$, we needed $\frac{n}{2}$ edges to delete all the edges of $P_{n}$ so $P_{m}$ is remained. Therefore,

$$
\operatorname{ENR}\left(P_{n}+P_{m}\right)=\left\{\begin{aligned}
\operatorname{ENR}\left(P_{m}\right)-\frac{n}{2}, & n \text { is even } \\
\operatorname{ENR}\left(P_{m-1}\right)-\left\lceil\frac{n}{2}\right\rceil, & n \text { is odd }
\end{aligned}\right.
$$

Case 2: If we select an edge-cut-strategy from combining edges of $P_{n}$ and $P_{m}$, We get,

$\operatorname{ENR}\left(P_{n}+P_{m}\right)=\operatorname{ENR}\left(P_{m-n}\right)-n$. 
Applied Mathematics and Sciences: An International Journal (MathSJ ), Vol. 5, No. 1/2/3, September 2018

Case 3:If we select an edge-cut-strategy from $P_{m}$, we need $\frac{n}{2}$ edges to delete all the edges of $P_{m} \mathrm{so} P_{n}$ is remained. Therefore,

$\operatorname{ENR}\left(P_{n}+P_{m}\right)=\left\{\begin{aligned} \operatorname{ENR}\left(P_{n}\right)-\frac{m}{2}, & m \text { is even } \\ \operatorname{ENR}\left(P_{n-1}\right)-\left\lceil\frac{m}{2}\right\rceil, & m \text { is odd }\end{aligned}\right.$

The results take maximum value in case 1 . So the proof is completed.

Corollary 2.3: The edge-neighbor-rupture degree of $P_{2}+P_{n}$ is,

$\operatorname{ENR}\left(P_{2}+P_{m}\right)=\operatorname{ENR}\left(P_{n}-1\right)$.

Theorem 2.5: $\operatorname{Let} G_{1}, G_{2}, \ldots, G_{n}$ be connected graphs then,

$\operatorname{ENR}\left(G_{1} \cup G_{2} \cup \ldots \cup G_{n}\right) \geq \operatorname{ENR}\left(G_{1}\right)+\operatorname{ENR}\left(G_{2}\right)+\cdots+\operatorname{ENR}\left(G_{n}\right)$

Proof: Let $G=G_{1} \cup G_{2} \cup \ldots \cup G_{n}$ be union of $G_{1}, G_{2}, \ldots, G_{n}$. Let $S=S_{1}, S_{2}, \ldots, S_{n}$ be an edge Nrset of $G_{1}, G_{2}, \ldots, G_{n}$ respectively and let $S=S_{1} \cup S_{2} \cup \ldots \cup S_{n}$ be an edge-subversion strategy of $G$. Then we obtain,

$$
\begin{aligned}
& \begin{aligned}
& \operatorname{ENR}(G) \geq \omega(G-S)-|S|-m(G-S) \\
& \quad=\left[\omega\left(G_{1}-S_{1}\right)+\omega\left(G_{2}-S_{2}\right)+\cdots+\omega\left(G_{n}-S_{n}\right)\right]-\left[\left|S_{1}\right|+\left|S_{2}\right|+\cdots+\left|S_{n}\right|\right] \\
&- {\left[m\left(G_{1}-S_{1}\right)+m\left(G_{2}-S_{2}\right)+\cdots+m\left(G_{n}-S_{n}\right)\right] } \\
&=\omega\left(G_{1}-S_{1}\right)-\left|S_{1}\right|-m\left(G_{1}-S_{1}\right)+\omega\left(G_{2}-S_{2}\right)-\left|S_{2}\right|-m\left(G_{2}-S_{2}\right)+\cdots+\omega\left(G_{n}-S_{n}\right) \\
&-\left|S_{n}\right|-m\left(G_{n}-S_{n}\right)
\end{aligned} \\
& \operatorname{ENR}\left(G_{1}\right)+\operatorname{ENR}\left(G_{2}\right)+\cdots+\operatorname{ENR}\left(G_{n}\right) .
\end{aligned}
$$

Theorem 2.6: Let $G$ be a connected graph, then the edge-neighbor-rupture degree of $G$ is,

$\operatorname{ENR}(G) \geq-\left\lfloor\frac{n}{2}\right\rfloor$

Proof: For the minimum value of $E N R(G), \omega(G-S)$ must be the smallest, $|S|$ and $m(G-S)$ must be the greatest.

Let $S$ be an edge-cut-strategy of $G$ and $|S|=r$. There are two cases for the elements of $S$.

Case 1: If $r \leq\left\lfloor\frac{n-1}{2}\right\rfloor$ then $\omega(G-S)=1$ and $m(G-S) \geq n-2 r$.

So we have

$\omega(G-S)-|S|-m(G-S) \leq 1-r-(n-2 r) \quad=1+r-n=f(r)$.

This equality takes maximum value for $r=\left\lfloor\frac{n-1}{2}\right\rfloor$.

There are two conditions:

Case i: If $n$ is odd; 
Applied Mathematics and Sciences: An International Journal (MathSJ ), Vol. 5, No. 1/2/3, September 2018

$f(r)=1+\frac{n-1}{2}-n=-\frac{n-1}{2}=-\left\lfloor\frac{n}{2}\right\rfloor$.

Case ii: If $n$ is even;

$f(r)=1+\frac{n-2}{2}-n=-\frac{n}{2}$

Case 2: If $\frac{n(n-1)}{2}>r>\left\lfloor\frac{n-1}{2}\right\rfloor$ then $\omega(G-S) \leq 1$ and $m(G-S) \geq 1$.

$\omega(G-S)-|S|-m(G-S) \leq 1-r-1=-r$.

This equality takes the maximum value for $r=\left\lfloor\frac{n}{2}\right\rfloor$.

From all cases, we obtain, $\operatorname{ENR}(G) \geq-\left\lfloor\frac{n}{2}\right\rfloor$.

Corollary 2.4: Let $G$ be a connected graph, then the edge-neighbor-rupture degree of $G$ is,

$-\left\lfloor\frac{n}{2}\right\rfloor \leq \operatorname{ENR}(G) \leq n-4$.

Proof: From Theorem 2.6 and [2], the proof is complete.

\section{Computing Edge-Neighbor-Rupture Degree OF a GraPH}

In this section, an algorithm is proposed in order to calculate the edge-neighbor-rupture degreefor any simple finite undirected graph without loops and multiple edges by using the findENR function.

Algorithm Edge Neighbor Rupture (ENR)

Output: ENR value for given any graph $G$

Begin

$\mathrm{ENR} \leftarrow-\infty$;

for all edge subsets $E_{S} \subseteq E$ do

if findENR $\left(G, E_{S}\right)>\operatorname{ENR}$ then

$\mathrm{ENR}=$ findENR $\left(G, E_{S}\right)$;

end

end

end.

The function below,find ENR, returns the ENR value for the edge subset for the graph.

function findENR $\left(G, E_{S}\right)$;

Input: $\operatorname{Graph} G(V, E)$, edgesubset $E_{S}$

Output:ENR value for given an $E_{S}$ edge subset of $G$.

Begin

$V_{S}$ : vertex set incident with $E_{S}$ edges.

for all $u \in V_{S}$ do

remove $u$ from $G\left\{\right.$ i.e. $\left.G-V_{s}\right\}$

end 
Applied Mathematics and Sciences: An International Journal (MathSJ ), Vol. 5, No. 1/2/3, September 2018

Componentnumber $\leftarrow$ find the number of components of $G-V_{S}$

MaxCompVertexnum $\leftarrow$ find the vertex number of maximum component of $G-V_{S}$

findENR $\leftarrow$ Component number - number of $\left(E_{S}\right)-$ MaxCompVertexnum $\}$

end

\section{CONCLusion}

In this study, we investigate the edge-neighbor rupture degree of graphs obtained by graph operations. The graph operations are used to obtain new graphs. Union, join and mostly thorny operations are taken into consideration in this work. These operations are performed to various graphs and their edge-neighbor rupture degrees were determined.

\section{REFERENCES}

[1] S. Kandilci, G. Bacak-Turan\&R. Polat, (2013), "Graph Operations and Neighbor Rupture Degree", Journal of Applied Mathematics, Hindawi Publishing Corporation, Article ID 836395, http://dx.doi.org/10.1155/2013/836395.

[2] E.Aslan, (2013), "Edge-Neighbor-Rupture Degree of Graphs", Journal of Applied Mathematics,Hindawi Publishing Corporation.Article ID 783610.

[3] C.A. Barefoot, R. Entringer\&H. Swart, (1987), "Vulnerability in graphs -Acomparative survey", Journal of Combinatorial Mathematics and Combinatorial Computing, vol1,pp 13-22.

[4] F. Buckley \& F. Harary, (1990), Distance in Graphs, Addision-Wesley Press.

[5] G. Chartrand \& L. Lesniak, (2005), Graphs \& Digraphs, Chapman \& Hall/CRC Press.

[6] I. Gutman, (1998), "Distance of Thorny Graphs", Publ. Institut Math. Nouvelle serie, vol63,pp 31-36.

[7] J. A. Bondy \& U. S. R.Murty, (1976), GraphTheory with Applications, The Macmillan Press.

[8] H. A. Jung, (1978), "On a class of posets and the corresponding comparability graphs",Journal of Combinatorial Theory B, vol 24,pp 125-133.

[9] Y. Li, S. Zhang \& X. Li, (2005), Rupture degree of graphs, International Journal of Computer Mathematics, vol 82,pp 793-803.

[10] M. B. Cozzens \& S. S.Y. Wu, (1998), "Vertex-neighbor-integrity ofpowers of cycles",Ars Combinatoria, vol 48, pp257-270.

[11] M. Saheli, H. Saati\&A. R. Ashrafi,(2010), "The eccentric connectivity index of one pentagonal carbon nanocones", Optoelectronics and Advanced Materials-Rapid Communications, vol4,pp 896897.

[12] M. Ghorbani,(2010), "GA Index of TUC4C8(R) Nanotube", Optoelectronics and Advanced Materials - Rapid Communications, vol 4, pp261-263.

[13] G. Bacak Turan \& A. Kirlangic, (2010), "Vulnerability Parameters in Graphs" 1st International Symposium onComputing in Science and Engineering, Turkey.

[14] M.Ü. Gürsoy,R. Polat, S. Eskiizmirliler\&Z.Ö. Yorgancığlu (2014), "Edge-Neighbor-Rupture-Degree on graph operations",9th AnkaraMath days, Atılım University, Ankara, Turkey. 
Applied Mathematics and Sciences: An International Journal (MathSJ ), Vol. 5, No. 1/2/3, September 2018

\section{AUTHORS}

Saadet Eskiizmirliler She was graduated fromYaşar University Mathematics department in 2010, she had got master degree in 2012 fromYaşar University Math department and she has been doing doktorate at Yaşar University Math department at applied mathematics since 2014. She has been working at Yasar University since 2014.

Zeynep Örs Yorgancioğlu She was graduated from Ege University Mathematics department in 2003, she had got master degree in 2010 from Ege University Math department and she had got $\mathrm{Ph}$. D degree in 2015 from Ege University Math department at applied Mathematics. She has been working at Yasar University since 2007.

Refet Polat Achieved his BSc degree in mathematics majoring in computer science at Ege University in 2000. He received his $\mathrm{MSc}$ and $\mathrm{PhD}$ degrees in applied mathematics at Ege University in 2003 and 2009. His research interests focus on applied mathematics, graph theory, ordinary differential equations, numerical methods, and artificial intelligence in education
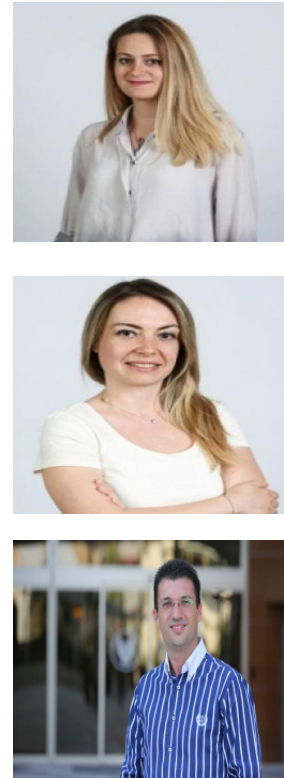

Mehmet Ümit Gürsoy $\mathrm{He}$ was graduated from Ege University Mathematics department in 2000, he had got master degree in 2005 from Ege University Math department and he had got Ph.D degree in 2014 from Ege University Math department at applied mathematics.

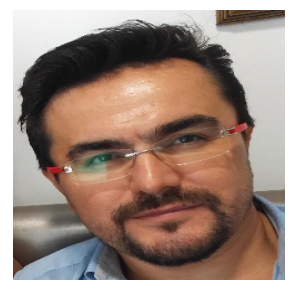

